\title{
COMPARACIÓN DE PROPIEDADES MECÁNICAS EN ALAMBRE DE ACERO CORRUGADO, DETERMINADAS MEDIANTE EL USO DE EXTENSÓMETRO DE CONTACTO Y EXTENSÓMETRO LÁSER
}

\author{
COMPARISON OF MECHANICAL PROPERTIES OF DEFORMED \\ STEEL WIRE DETERMINED USING CONTACT AND LASER \\ EXTENSOMETERS
}

Fecha de recepción: 02 de Septiembre de 2015

Ing. Ana Lorena Monge Sandí, M.Sc

Investigadora Programa de Ingeniería Geotécnica

LanammeUCR, Costa Rica

E-mail: ana.mongesandi@ucr.ac.cr
Fecha de aceptación: 08 de Enero de 2016

Ana María Rodríguez Pereira

Estudiante Ingeniería en Materiales

Tecnológico de Costa Rica, Costa Rica

E-mail: ani07amrp@gmail.com

\section{RESUMEN}

En esta investigación se presenta el estudio de 16 muestras de alambres de acero, a las cuales se les aplicó un ensayo de tensión y cuyas deformaciones fueron medidas utilizando dos tipos de extensómetro: láser y de contacto. El objetivo principal del análisis es definir si los resultados recuperados del extensómetro láser presentan la suficiente precisión como para determinar propiedades mecánicas adecuadamente, es decir validar el uso del extensómetro láser en el ensayo de tensión; así como generar una metodología de estudio para materiales con región de fluencia no definida. A partir de los resultados obtenidos, se concluye que el extensómetro láser produce datos cuantitativamente similares a los del de contacto, por lo que se valida el uso del equipo para el ensayo de tensión; además se confirma que la metodología desarrollada permite calcular de manera apropiada las propiedades mecánicas de materiales con región de fluencia no definida.

\section{PALABRAS CLAVE:}

Extensómetro de contacto, extensómetro láser, esfuerzo de fluencia, alambre para refuerzo.

\section{ABSTRACT}

This research paper presents the study of 16 samples of deformed steel wires, which were applied to tensile tests using two types of extensometers: contact and laser. The main objective of the analysis is to determine if the results obtained by the laser extensometer have sufficient accuracy to determine the tensile mechanical properties properly, in order to validate the use if laser extensometer in standard laboratory tensile tests, as well as determine a methodology of the obtainment of the mechanical properties of steel samples with an undefined yield point. From the results, it is concluded that the laser extensometer, as well as the methodology used, produce results which are quantitative similar to the contact extensometer, therefore validating its use for standard laboratory tensile testing of materials with an undefined yield point.

\section{KEY WORDS:}

Conventional extensometers, laser extensometer, yield strength, reinforcement wire. 


\section{INTRODUCCIÓN}

El alambre de acero es usado a nivel comercial como refuerzo para estructuras de concreto, por parte de la industria de la construcción. Esta industria debe darle gran importancia a la seguridad, por lo que existe una necesidad permanente de garantizar la calidad del material por utilizar en la obra.

Debido a esto, se han desarrollado a lo largo de los años métodos de análisis que permiten determinar la calidad del material a partir de las propiedades mecánicas, químicas y físicas.

Esta necesidad es incluso reflejada a nivel nacional, mediante la creación e implementación del Reglamento Técnico para Barras y Alambres de Acero de refuerzo para concreto (Reglamento Técnico RTCR 452:2011 Barras y Alambres de Acero de refuerzo para concreto, 2013).

Uno de los principales ensayos utilizados para determinar la calidad del material es el ensayo de esfuerzo-deformación, el cual consiste en someter la muestra a un esfuerzo axial a partir del cual se producen deformaciones unitarias, de las cuales se genera una gráfica que es comparada con una curva característica del material (Avner, 1988).

Para realizar este ensayo se utilizan equipos especializados, como los extensómetros de contacto de puntas. Estos consisten en dos puntas metálicas que se ponen en contacto con la muestra a una distancia controlada, y conforme se aplica la carga de deformación, el equipo registra la elongación generada por la separación entre las puntas (ASTM E83).

Los extensómetros de contacto presentan una alta sensibilidad, producto del contacto físico entre las dos puntas metálicas y la muestra. Este contacto físico produce que no sea posible utilizar este equipo para realizar mediciones de las propiedades mecánicas en el rango total de análisis, es decir, hasta la ruptura de la muestra, pues el impacto producido por la ruptura podría generar un daño permanente sobre el equipo.

Con el fin de solventar esta falencia, en el mercado se ha buscado implementar el uso de equipos de medición que permitan realizar el análisis en el rango completo, como es el extensómetro láser. Este equipo, a diferencia del de contacto, no requiere un contacto físico para el análisis, lo que permite el análisis de propiedades mecánicas en la totalidad del ensayo.

El equipo del extensómetro láser consiste en dos componentes principales, un emisor de rayos láser y un sensor que recibe la señal desviada (MTS Systems Corporation, 2009). Su funcionamiento es simple: se coloca cinta retroreflectiva en los límites de la longitud de control, el equipo emite el rayo láser, y el sensor recibe la señal reflejada. A partir de la diferencia entre el rayo inicial y final, se calcula la deformación unitaria.
A pesar de que el extensómetro láser tiene la ventaja de que permite realizar el ensayo con el equipo hasta ruptura, este equipo cuenta con el inconveniente de presentar una resolución menor que el extensómetro de contacto, pues ésta es de $0,000001 \mathrm{~mm} / \mathrm{mm}$ contra la resolución del láser que es de $0,0005 \mathrm{~mm}$.

Dado que el extensómetro de contacto no permite el análisis de propiedades mecánicas hasta ruptura, y que al utilizar el extensómetro láser hay una disminución en la precisión de la medición, el objetivo principal de esta investigación es validar mediante un estudio estadístico si las mediciones de deformaciones unitarias en alambre de acero, obtenidas con el extensómetro láser, reproducen datos lo suficientemente confiables, comparables con los del extensómetro de contacto.

Para esta investigación, se parte de la metodología aplicada en el estudio realizado por Monge y Abarca (2013); pero únicamente en los parámetros del equipo, para obtener los datos de ambos extensómetros; ya que el procedimiento utilizado para calcular las propiedades mecánicas cambia.

El material base para esta investigación es alambre de acero que puede ser utilizado como refuerzo para concreto. Este es un alambre de acero al Carbono conformado por laminación en caliente hasta obtener las corrugaciones según la ASTM A1064. Es decir, los lingotes de metal se someten a presión y temperatura por medio de rodillos hasta formar las alambres y posteriormente, se le realizan las corrugaciones a alta presión y bajas temperaturas (Smith y Hashemi, 2006).

Para el estudio se utilizaron muestras de dos diámetros diferentes: $5,25 \mathrm{~mm}$ y $6,35 \mathrm{~mm}$. Las muestras de $5,25 \mathrm{~mm}$ presentan una aleación de bajo Carbono, con $0.12 \%$, aleada con Manganeso, Silicio, y Azufre; mientras que las de 6,35 $\mathrm{mm}$ presenta los mismos aleantes, pero en $0,05 \%$ Carbono (especificaciones dadas por el fabricante).

El criterio de escogencia de la muestra se basó en la importancia y el uso del material a nivel comercial, pero principalmente a raíz de su comportamiento en el diagrama esfuerzo-deformación unitaria. Esto se debe a que el alambre por analizar no presenta una región definida de fluencia, por lo que se debe obtener el esfuerzo de fluencia de manera numérica, dificultando su análisis.

A partir de lo anterior, se recalca la importancia de esta investigación. Inicialmente por la necesidad de conocer la aplicabilidad del extensómetro láser para la determinación de propiedades mecánicas en alambres de acero; y además, que a partir de esta investigación surge un procedimiento de análisis para una materia prima de importancia comercial, cuyas propiedades mecánicas son difíciles de determinar.

De esta manera, esta investigación tiene como principales alcances: medir deformaciones unitarias en varillas corrugadas para refuerzo de concreto de diámetro $5,25 \mathrm{~mm}$ y 
$6,35 \mathrm{~mm}$. A partir de las deformaciones medidas, calcular las propiedades mecánicas de esfuerzo de fluencia y esfuerzo de ruptura, mediante el método de extensión bajo carga (ASTM A 1064). Lo anterior se realiza para dos tipos de extensómetro: láser y de contacto. $\mathrm{Y}$ por último, con base en los valores de las propiedades mecánicas calculadas, definir si los resultados obtenidos usando del extensómetro láser, reproducen la suficiente confiabilidad como para ser comparados con las mediciones obtenidas con el extensómetro de contacto.

\section{OBJETIVO}

Determinar la validez de la utilización de los datos provenientes del extensómetro láser con que cuenta el LanammeUCR para obtener los parámetros mecánicos de los alambres de refuerzo para concreto regidos bajo las especificaciones de la norma ASTM A1064, comparando los resultados con los obtenidos utilizando un extensómetro de contacto.

\section{MATERIALES Y METODOLOGÍA}

\subsection{Descripción del Equipo}

Para realizar el ensayo de tensión se utilizan tres equipos principalmente: la máquina de esfuerzo universal Landmark, extensómetro de contacto y láser; los tres fabricados por la empresa MTS Systems Corporations. A continuación se presentan las especificaciones de cada equipo.

\subsubsection{Máquina universal}

Se utiliza el modelo Landmark con una capacidad de $25 \mathrm{kN}$.

\subsubsection{Extensómetro de contacto}

Se utiliza un extensómetro modelo 634.25F-24, el cual tiene una longitud interna de galga de $50 \mathrm{~mm}$ que permite un rango de medición de $25 \mathrm{~mm}$ en tensión a $-5 \mathrm{~mm}$ en compresión, cumpliendo con la especificación indicada en la norma ASTM E83 para extensómetros clase B1.

\subsubsection{Extensómetro láser}

Se utiliza el equipo modelo LX-1500. El extensómetro presenta una amplitud de medición de $381( \pm 0,01) \mathrm{mm}$, cumpliendo con la especificación indicada en la norma ASTM E83 para extensómetros clase B1.

\subsection{Descripción de la Muestra}

Para este estudio, se utilizaron 16 alambres grafilados de acero aleado con bajo contenido de carbono, de dos diámetros y grados diferentes (Tabla 1 y Figura 1), aunque para cada diámetro se contaba en total con 15 especímenes. Las muestras para cada diámetro son del mismo lote.

\begin{tabular}{|c|c|c|c|c|c|c|c|}
\hline $\begin{array}{c}\text { Codificación } \\
\text { de la Muestra }\end{array}$ & $\begin{array}{c}\text { Norma } \\
\text { ASTM }\end{array}$ & $\begin{array}{c}\text { Diámetro } \\
\text { indicado } \\
\text { por el } \\
\text { fabricante } \\
(\mathbf{m m})\end{array}$ & $\begin{array}{c}\text { Diámetro } \\
\text { Nominal* } \\
(\mathbf{m m})\end{array}$ & $\begin{array}{c}\text { Área } \\
\text { nominal } \\
(\mathbf{m m})\end{array}$ & Grado** & $\begin{array}{c}\text { Cantidad } \\
\text { Longitud de } \\
\text { la muestra } \\
(\mathbf{m m})\end{array}$ \\
\hline M.2267-14 & $\begin{array}{c}\text { ASTM } \\
\text { A1064M }\end{array}$ & 5,25 & 5,05 & 20 & 40 & 8 & 385 \\
\hline M.2268-14 & $\begin{array}{c}\text { ASTM } \\
\text { A1064M }\end{array}$ & 6,35 & 6,18 & 30 & 70 & 8 & 385 \\
\hline
\end{tabular}

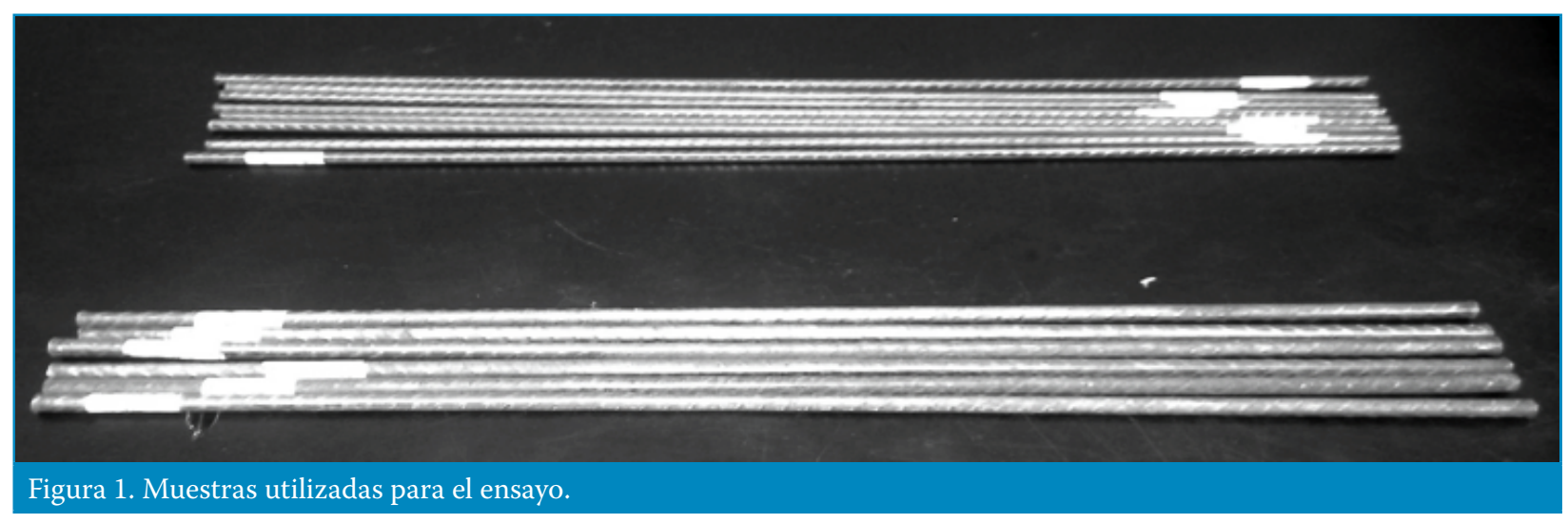




\subsection{Configuración del Ensayo e Instrumentación}

Debido a que en el LanammeUCR se aplica un procedimiento estandarizado en el estudio de propiedades mecánicas de materiales de construcción, para la investigación se utilizó la metodología de la preparación de la muestra, el ensayo de tensión y la recuperación de datos, aplicada en el estudio "Comparación de determinación de propiedades mecánicas en barras de refuerzo para concreto con extensómetro de contacto y extensómetro láser" (Monge y Abarca, 2013). Esto debido a que, a pesar de que los alambres presentan diferentes diámetros y grados, el procedimiento para estudiar sus propiedades debe ser estandarizado con el fin de garantizar resultados fiables.

La metodología utilizada consistió de cuatro partes: la preparación de la muestra, cálculo del diámetro nominal, la aplicación del ensayo de tensión y por último la determinación matemática de las propiedades mecánicas. La preparación de la muestra y la aplicación del ensayo de tensión siguen los parámetros utilizados en el estudio previamente mencionado.

\subsubsection{Preparación de las muestras}

Para preparar las muestras, se calculó la longitud total de acuerdo a lo establecido a la norma ASTM A 370. El cálculo consistió en la suma de la longitud de control predefinida como $200 \mathrm{~mm}$, más dos veces el diámetro más $85 \mathrm{~mm}$ por cada mordaza. Resultando en una longitud final de $385 \mathrm{~mm}$.

\subsubsection{Cálculo del diámetro nominal}

Los alambres analizados presentan dos diámetros diferentes: $5,25 \mathrm{~mm}$ y $6,35 \mathrm{~mm}$. Estas dimensiones no se adaptan a los valores de diámetro nominal preestablecidos por la norma ASTM A1064, de manera que se implementó lo indicado en esta misma para determinarlo.

Para calcular el diámetro nominal se determina inicialmente el área nominal. Ésta se obtiene al despejar su valor a partir de la densidad y los valores de masa y longitud de las muestras, previamente medidos. Una vez obtenido el valor, se redondea a la decena más cercana. Con el valor de área nominal, se despeja de la fórmula del área, el valor del diámetro nominal.

\subsubsection{Ensayo de tensión}

El proceso del ensayo de tensión consistió en:

- Colocar las cintas retroflectivas a una distancia de análisis de $200 \mathrm{~mm}$ sobre las muestras (cabe aclarar que la longitud de control de $200 \mathrm{~mm}$ es la misma para los dos tipos de extensómetros)

- Montar la muestra en la máquina

- Colocar el extensómetro de contacto en la muestra y el láser frente al equipo

- Asignar el valor de velocidad de carga
- Aplicar la carga

- Respaldar los resultados del ensayo

\subsubsection{Cálculo de propiedades mecánicas}

Para determinar las propiedades mecánicas a partir del ensayo de tensión, se utiliza el método gráfico de extensión bajo carga con la deformación especificada (ASTM A 370). En esta investigación se calcularon dos propiedades mecánicas: esfuerzo de fluencia y esfuerzo máximo; dos cambios físicos: reducción de área, elongación y el Módulo de elasticidad.

La determinación del esfuerzo de fluencia se hizo graficando una línea vertical en el punto de deformación unitaria de $0,005 \mathrm{~mm} / \mathrm{mm}$, el cual está definido por la norma de especificación del alambre, ASTM A 1064 (Ver Figura 2). Una vez trazada la línea, se busca el punto de intersección de ésta con la curva Esfuerzo-Deformación Unitaria, y el valor de esfuerzo de fluencia es el correspondiente a esta intersección en el eje de las ordenadas.

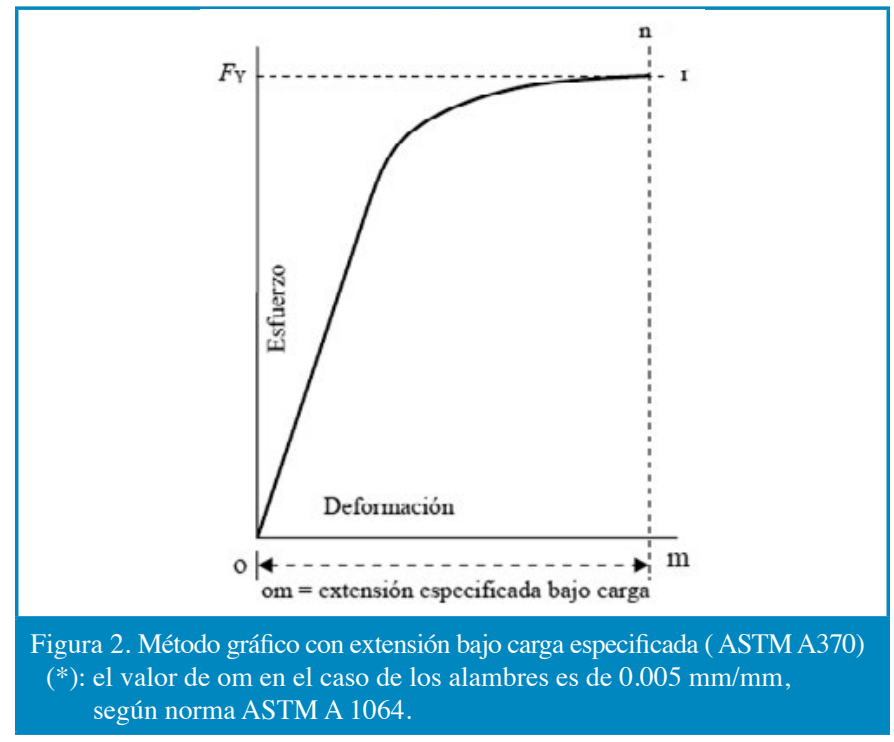

Por su parte, el esfuerzo máximo se calculó como el mayor valor de esfuerzo obtenido de la gráfica de EsfuerzoDeformación Unitaria. Para el cálculo de ambos valores, el esfuerzo de fluencia y máximo, se divide el valor de carga dado por la máquina en el punto de análisis, entre el área nominal definida anteriormente.

Para determinar el valor del Módulo de elasticidad se escogió un rango donde existiera proporcionalidad lineal en la curva esfuerzo-deformación unitaria, en este caso de 100 a 300 $\mathrm{MPa}$. El valor de la pendiente de la gráfica para ambos tipos de extensómetros, corresponden al valor del Módulo de elasticidad.

El cálculo de elongación máxima se realizó mediante dos procedimientos: numérico y físico, ambos descritos por la norma ASTM A370. El método físico consistió en medir 
la distancia final entre las marcas de la longitud de control (ver figura 3). Y a partir del valor inicial y final calcular la elongación final de acuerdo a la siguiente fórmula:

$$
\sigma_{\operatorname{máx}}=\frac{l_{i}-l_{f}}{l_{i}} * 100 \quad[1]
$$

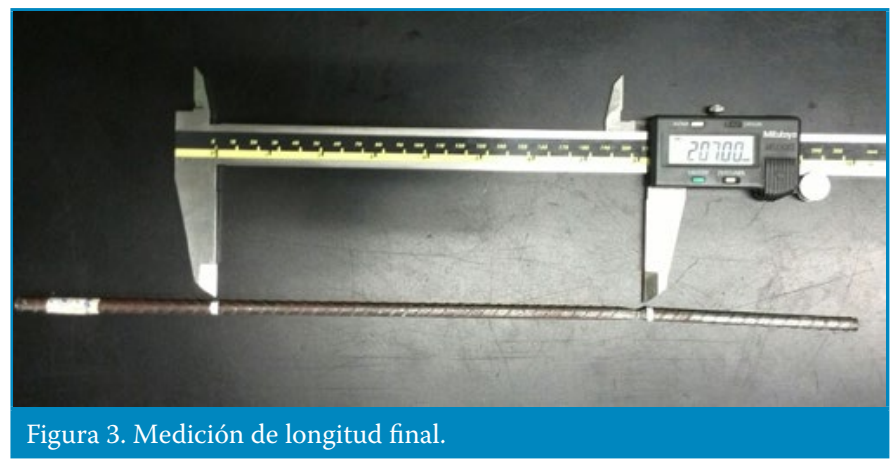

Las curvas realizadas a partir del ensayo de tensión describen dos tipos de forma: una caída progresiva a partir del punto de esfuerzo máximo (Figura 4.a), o una caída repentina en forma de la letra "L" (Figura 4.b). A partir de esta diferencia, la norma ASTM A370 determina que el esfuerzo máximo debe calcularse de manera diferenciada para cada tipo de forma.

Debido a esto, el método analítico para el cálculo del esfuerzo máximo se realizó de dos maneras, de acuerdo con el tipo de forma que presentó la gráfica. Si esta presenta una caída en forma de la letra "L", el valor de elongación máxima se estableció de manera directa (Figura 4.a). Si la gráfica presenta una caída gradual a partir del valor de esfuerzo máximo (Figura 4.b), se grafica una recta a $10 \%$ por debajo del esfuerzo máximo; y el valor de deformación unitaria se define como en la intersección entre la recta y la gráfica de esfuerzodeformación unitaria. La elongación es esta deformación unitaria expresada en porcentaje.

Por último, el valor de esfuerzo de fluencia, para cada tipo de extensómetro, se determinó a partir de los datos reportados por la máquina. Esto debido a que el equipo utilizado para recuperar los datos reporta los valores de esfuerzo y deformación como pares ordenados, es decir, para cada valor de esfuerzo, el equipo reporta un valor de deformación asociado a este esfuerzo.

\subsection{Análisis Estadístico}

Se realizó un análisis estadístico con el fin de definir si existe una diferencia significativa (al 95\% de nivel de confianza) entre los datos obtenidos mediante la medición con el extensómetro láser y el de contacto. El análisis estadístico se aplicó a los parámetros de Módulo de elasticidad, esfuerzo máximo y esfuerzo de fluencia.

Para la metodología aplicada para este estudio se realizó un análisis de varianza de un grado de libertad, mediante la prueba de Fisher. Se identificaron los parámetros de interés, presentados en la Tabla 2.

\begin{tabular}{|c|l|}
\hline Parámetro & \multicolumn{1}{|c|}{ Descripción } \\
\hline$\varepsilon_{\mathrm{u}(\text { num })}$ & Elongación máxima medida por el método numérico. \\
\hline$\varepsilon_{\mathrm{u}(\mathrm{fis})}$ & Elongación máxima medida por el método físico. \\
\hline $\mathrm{E}_{\text {(ext) }}$ & $\begin{array}{l}\text { Módulo de elasticidad calculado a partir de datos del } \\
\text { extensómetro laser. }\end{array}$ \\
\hline $\mathrm{E}_{\text {(laser) }}$ & $\begin{array}{l}\text { Módulo de elasticidad calculado a partir de datos del } \\
\text { extensómetro de contacto. }\end{array}$ \\
\hline $\mathrm{F}_{\text {(conv) }}$ & $\begin{array}{l}\text { Esfuerzo de fluencia obtenido mediante el } \\
\text { extensómetro de contacto }\end{array}$ \\
\hline $\mathrm{F}_{\mathrm{y}(\text { laser })}$ & $\begin{array}{l}\text { Esfuerzo de fluencia obtenido mediante el } \\
\text { extensómetro láser }\end{array}$ \\
\hline
\end{tabular}

Tabla 2. Parámetros de interés para determinar si existen diferencias significativas del uso del Extensómetro láser para la obtener propiedades mecánicas, a partir de deformaciones unitarias

Luego se establecieron las hipótesis nulas a evaluar mediante el análisis estadístico, definidas en la Tabla 3.

\begin{tabular}{|c|l|}
\hline Parámetro & \multicolumn{1}{|c|}{ Descripción } \\
\hline$\varepsilon_{\mathrm{u}(\text { num })}$ & Elongación máxima medida por el método numérico. \\
\hline$\varepsilon_{\mathrm{u}(\mathrm{fis})}$ & Elongación máxima medida por el método físico. \\
\hline $\mathrm{F}_{\mathrm{y}(\text { láser })}$ & $\begin{array}{l}\text { Esfuerzo de fluencia obtenido mediante el } \\
\text { extensómetro láser }\end{array}$ \\
\hline
\end{tabular}

Tabla 2. Parámetros de interés para determinar si existen diferencias significativas del uso del Extensómetro láser para la obtener propiedades mecánicas, a partir de deformaciones unitarias

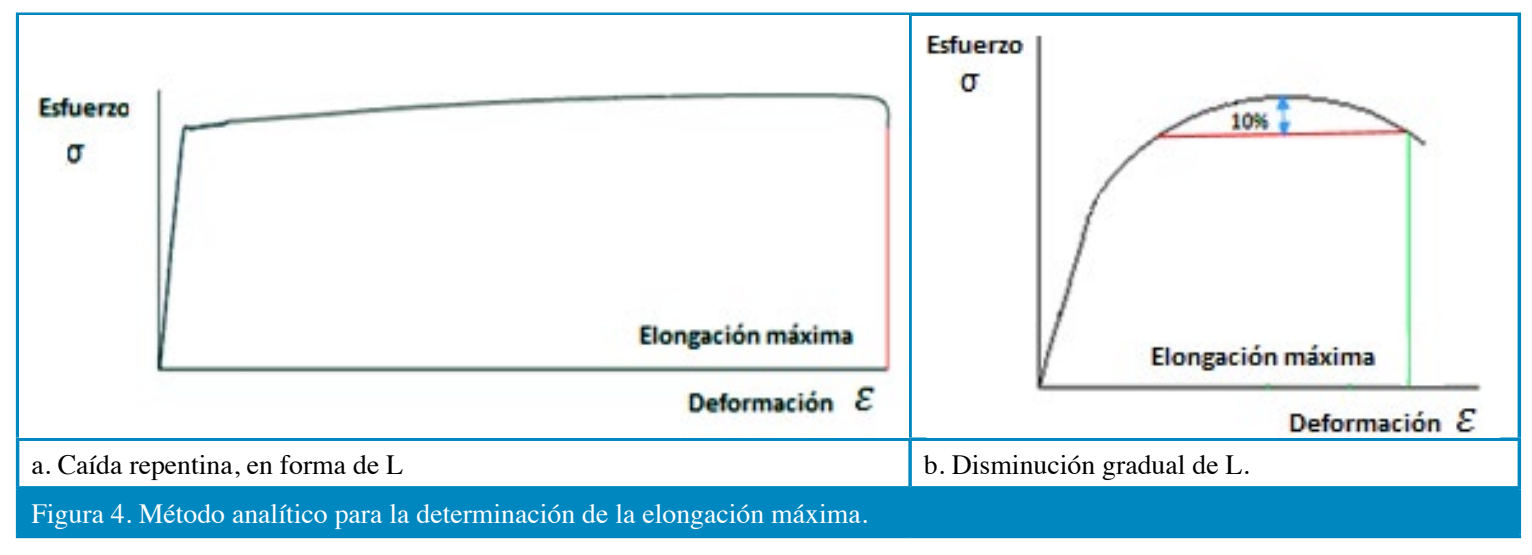


Posteriormente, se definió el nivel de confiabilidad como $95 \%$, es decir, un $\alpha=0,05 \%$. A partir de lo anterior, se calculó el parámetro $\mathrm{F}$ y se estableció el criterio de rechazo a partir de los valores teóricos obtenidos de la literatura (Navarro, 2010). Por último, se realizó la comparación entre el estadístico calculado y el criterio de rechazo, a partir de la cual se definió si la hipótesis nula es o no aceptada.

\section{ANÁLISIS Y RESULTADOS}

\subsection{Propiedades de las Alambres}

\subsubsection{Gráficos de Esfuerzo-Deformación Unitaria}

De acuerdo con el procedimiento descrito en la sección anterior se generaron gráficas Esfuerzo-Deformación Unitaria para cada muestra. A partir de estas se obtuvieron los valores de esfuerzo de fluencia y máximo, el Módulo de elasticidad y la elongación máxima. La gráfica presentada a continuación ejemplifica el método utilizado para la recuperación de los datos previamente mencionados.
La Figura 5 hace posible observar que ambos extensómetros reproducen un comportamiento cuantitativamente similar, tanto en la región de proporcionalidad lineal, como en la sección posterior dónde se calculó el valor de esfuerzo de fluencia.

Debido a que en esta investigación se pretende determinar que no existen diferencias significativas para los datos obtenidos por el extensómetro láser y que produce resultados fiables que permitan analizar propiedades mecánicas de las alambres, se esperó que todas las muestras analizadas presentaran un comportamiento similar al mostrado en la Figura 5.

\subsubsection{Determinación del Módulo de elasticidad}

Para determinar el valor de Módulo de elasticidad, tanto para extensómetro de contacto como láser, se graficó el rango de esfuerzo de 100 a $300 \mathrm{MPa}$, correspondiente a una parte de la zona de proporcionalidad lineal. El Módulo se obtuvo como la pendiente de la relación entre el esfuerzo y la deformación unitaria.

En la Figura 6 se presenta como ejemplo del
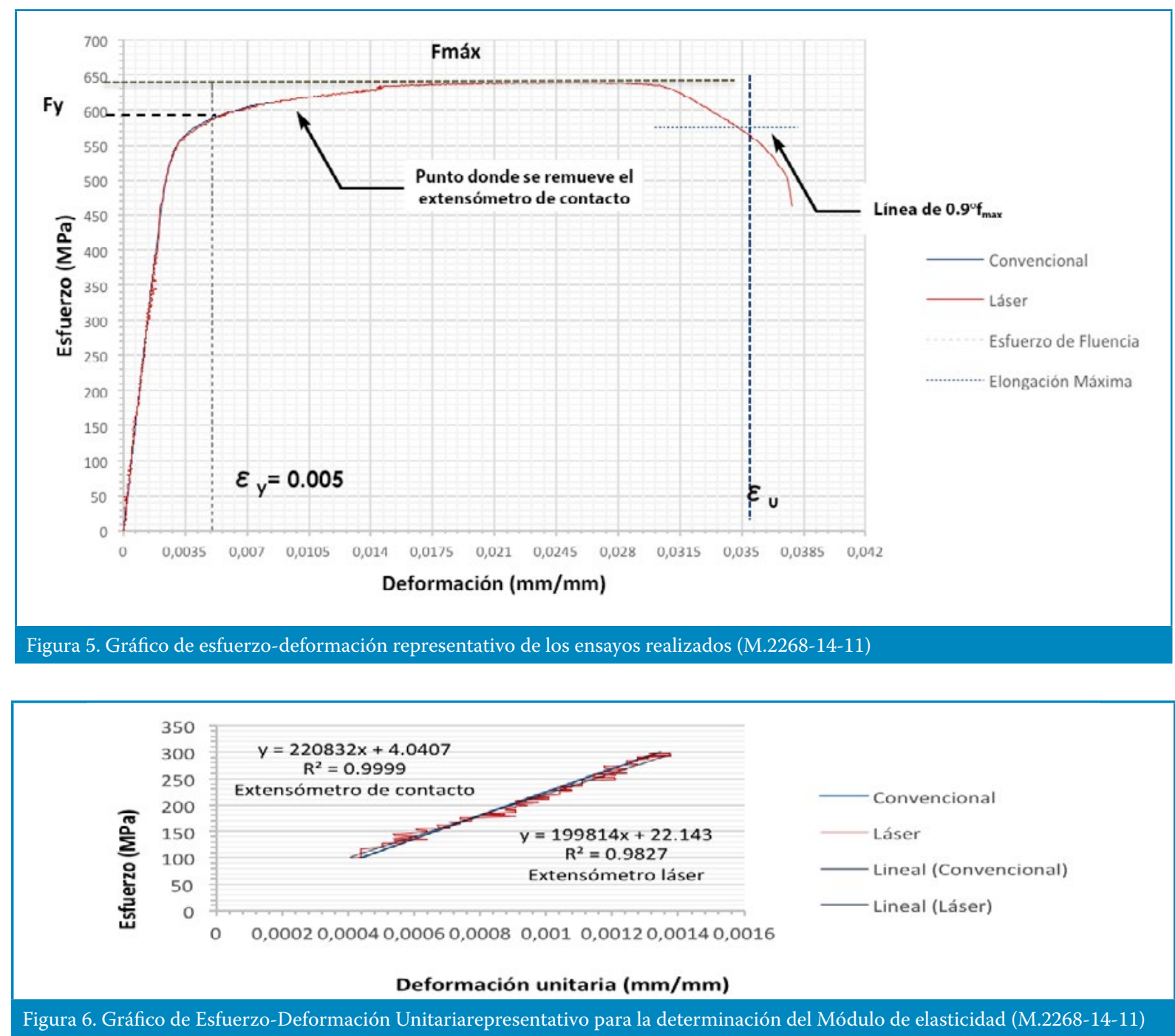
procedimiento de obtención de datos para una de las muestras analizadas. En esta se observa cómo, a pesar de que los datos producidos por el extensómetro láser tienen un coeficiente de correlación que genera menor confiabilidad, los valores de módulo de elasticidad presentan valores similares a los obtenidos con el extensómetro de contacto.

Al igual que en el análisis del diagrama esfuerzodeformación, se buscó que todas las muestras analizadas produjeran valores de módulos de elasticidad con diferencias poco significativas entre el extensómetro láser y el de contacto.

\subsubsection{Valores esfuerzo de fluencia y máximo}

Los resultados de esfuerzo de fluencia y máximo para las muestras de diámetro $5,25 \mathrm{~mm}$, resumidos en la tabla 4 , cumplen con las especificaciones mínimas establecidas por el fabricante, pues todas las muestras superan los valores de $320 \mathrm{MPa}$ de esfuerzo de fluencia y $480 \mathrm{MPa}$ de esfuerzo máximo. Cabe recordar que el esfuerzo máximo no depende del uso de alguno de los extensómetros.

De la misma manera, las muestras de diámetro $6,35 \mathrm{~mm}$, cuyos resultados se observan en la tabla 5 , cumplen con las especificaciones mínimas definidas por la norma ASTM A1064, que establece $515 \mathrm{MPa}$ como valor mínimo aceptable de esfuerzo de fluencia, y $585 \mathrm{MPa}$ para el esfuerzo último o de ruptura (ASTM A1064).

\begin{tabular}{|c|c|c|c|}
\hline \multirow{2}{*}{$\begin{array}{l}\text { Número } \\
\text { de muestra }\end{array}$} & \multirow{2}{*}{$\begin{array}{l}\text { Código } \\
\text { de muestra }\end{array}$} & $f_{y}$ & $\mathrm{~F}_{\max }$ \\
\hline & & $\begin{array}{l}\text { Esfuerzo } \\
\text { Fluencia }\end{array}$ & Esfuerzo Máximo \\
\hline 1 & M2267-14-1 & 458.61 & 503.60 \\
\hline 2 & M2267-14-5 & 455.68 & 509.18 \\
\hline 3 & M2267-14-6 & 428.36 & 485.22 \\
\hline 4 & M2267-14-8 & 465.24 & 520.97 \\
\hline 5 & M2267-14-9 & 386.31 & 435.49 \\
\hline 6 & M2267-14-11 & 447.87 & 500.87 \\
\hline 7 & M2267-14-12 & 440.19 & 493.90 \\
\hline 8 & M2267-14-13 & 460.21 & 510.02 \\
\hline & Promedio & 442.81 & 494.91 \\
\hline & sviación Estándar & 25.79 & 26.32 \\
\hline
\end{tabular}

\begin{tabular}{|c|c|c|c|}
\hline \multirow{2}{*}{$\begin{array}{l}\text { Número } \\
\text { de muestra }\end{array}$} & \multirow{2}{*}{$\begin{array}{l}\text { Código } \\
\text { de muestra }\end{array}$} & $f_{y}$ & $\mathrm{~F}_{\max }$ \\
\hline & & Esfuerzo Fluencia & Esfuerzo Máximo \\
\hline 1 & M2268-14-1 & 579.51 & 631.77 \\
\hline 2 & M2268-14-10 & 562.19 & 612.21 \\
\hline 3 & M2268-14-11 & 587.59 & 639.52 \\
\hline 4 & M2268-14-12 & 591.48 & 646.34 \\
\hline 5 & M2268-14-13 & 596.35 & 637.70 \\
\hline 6 & M2268-14-15 & 589.80 & 637.05 \\
\hline 7 & M2268-14-16 & 590.36 & 637.64 \\
\hline 8 & M2268-14-17 & 569.10 & 621.22 \\
\hline & Promedio & 583.30 & 632.93 \\
\hline & iación Estándar & 12.01 & 11.04 \\
\hline
\end{tabular}

\subsubsection{Valores Módulo de elasticidad}

Las Tablas 6 y 7 presentan los valores de Módulo de elasticidad obtenidos a partir de los resultados de cada extensómetro, para cada diámetro de alambre. A partir de éstas, al igual que en la Figura 5, se puede observar que los resultados presentan un comportamiento cuantitativamente similar. Esta hipótesis se comprobará mediante el análisis estadístico posterior.

(ver tabla 6 y 7 en pagina 33 ).

\subsubsection{Valores de elongación máxima}

En el caso de las Tablas 8 y 9, se presentan los valores de elongación máxima medidas por el método físico y gráfico. A diferencia de los resultados analizados en la curva de Esfuerzo-Deformación Unitaria completa, del análisis de la sección de proporcionalidad y de los Módulos de elasticidad para cada tipo de extensómetro, en este caso se presentan resultados con mayor variabilidad. (ver tabla 8 y 9 en pagina 33 ).

\subsection{Análisis estadístico}

\subsubsection{Análisis del valor elongación máxima}

Al aplicar la prueba de hipótesis nula a los resultados obtenidos de elongación máxima, se obtienen los siguientes resultados:

Al observar los resultados en la Tabla 10, se puede afirmar que la prueba de hipótesis nula no se rechaza. Esto implica que, a pesar de que los métodos de obtención de los valores de elongación máxima implican un error humano asociado, es posible afirmar con un $95 \%$ de nivel de confianza, que ambos métodos producen resultados cuantitativamente similares y por ende, no existen diferencias significativas en los resultados obtenidos. (ver tabla 10 en pagina 33 ). 


\begin{tabular}{|c|c|c|c|}
\hline \multirow[b]{2}{*}{$\begin{array}{c}\text { Número } \\
\text { de muestra }\end{array}$} & \multirow[b]{2}{*}{$\begin{array}{l}\text { Código } \\
\text { de muestra }\end{array}$} & $\mathrm{E}_{\text {conv }}(\mathrm{GPa})$ & \multirow{2}{*}{$\begin{array}{c}\mathbf{E}_{\text {láser }}(\mathbf{G P a}) \\
\text { Módulo de } \\
\text { Young Láser }\end{array}$} \\
\hline & & $\begin{array}{c}\text { Módulo } \\
\text { de Young } \\
\text { Convencional }\end{array}$ & \\
\hline 1 & M2267-14-1 & 205.91 & 219.32 \\
\hline 2 & M2267-14-5 & 216.17 & 189.77 \\
\hline 3 & M2267-14-6 & 214.76 & 215.61 \\
\hline 4 & M2267-14-8 & 212.41 & 215.80 \\
\hline 5 & M2267-14-9 & 193.67 & 196.72 \\
\hline 6 & M2267-14-11 & 211.50 & 216.69 \\
\hline 7 & M2267-14-12 & 212.38 & 204.59 \\
\hline 8 & M2267-14-13 & 220.10 & 207.53 \\
\hline & Promedio & 210.86 & 208.25 \\
\hline \multicolumn{2}{|c|}{ Desviación Estándar } & 8.05 & 10.65 \\
\hline
\end{tabular}

\begin{tabular}{|c|c|c|c|}
\hline \multirow[b]{2}{*}{$\begin{array}{l}\text { Número } \\
\text { de muestra }\end{array}$} & \multirow[b]{2}{*}{$\begin{array}{c}\text { Código } \\
\text { de muestra }\end{array}$} & $\mathrm{E}_{\text {conv }}(\mathrm{GPa})$ & $\mathrm{E}_{\text {láser }}(\mathrm{GPa})$ \\
\hline & & $\begin{array}{c}\text { Módulo } \\
\text { de Young } \\
\text { Convencional }\end{array}$ & $\begin{array}{l}\text { Módulo de } \\
\text { Young Láser }\end{array}$ \\
\hline 1 & M2268-14-1 & 220.01 & 199.91 \\
\hline 2 & M2268-14-10 & 214.72 & 184.49 \\
\hline 3 & M2268-14-11 & 220.83 & 199.81 \\
\hline 4 & M2268-14-12 & 213.17 & 164.22 \\
\hline 5 & M2268-14-13 & 203.61 & 215.12 \\
\hline 6 & M2268-14-15 & 216.84 & 195.30 \\
\hline 7 & M2268-14-16 & 211.58 & 185.58 \\
\hline 8 & M2268-14-17 & 206.49 & 202.18 \\
\hline & Promedio & 213.41 & 193.33 \\
\hline De & iación Estándar & 6.09 & 15.25 \\
\hline
\end{tabular}

\begin{tabular}{|c|c|c|c|}
\hline \multirow[b]{2}{*}{$\begin{array}{l}\text { Número } \\
\text { de muestra }\end{array}$} & \multirow[b]{2}{*}{$\begin{array}{l}\text { Código } \\
\text { de muestra }\end{array}$} & $\varepsilon_{\text {máx }}$ medida & \multirow{2}{*}{$\begin{array}{c}\boldsymbol{\varepsilon}_{\text {máx }} \text { láser } \\
\text { Elongación } \\
\text { máxima } \\
\text { por método } \\
\text { analítico }\end{array}$} \\
\hline & & $\begin{array}{l}\text { Elongación } \\
\text { máxima por } \\
\text { método físico }\end{array}$ & \\
\hline 1 & M2267-14-1 & 3.90 & 4.00 \\
\hline 2 & M2267-14-5 & 8.99 & 8.30 \\
\hline 3 & M2267-14-6 & 6.93 & 5.55 \\
\hline 4 & M2267-14-8 & 8.41 & 8.00 \\
\hline 5 & M2267-14-9 & 7.78 & 6.80 \\
\hline 6 & M2267-14-11 & 7.02 & 8.20 \\
\hline 7 & M2267-14-12 & 6.22 & 6.40 \\
\hline 8 & M2267-14-13 & 5.10 & 4.60 \\
\hline & Promedio & 6.79 & 6.48 \\
\hline \multicolumn{2}{|c|}{ Desviación Estándar } & 1.69 & 1.66 \\
\hline
\end{tabular}

\begin{tabular}{|c|c|c|c|}
\hline \multirow[b]{2}{*}{$\begin{array}{l}\text { Número } \\
\text { de muestra }\end{array}$} & \multirow[b]{2}{*}{$\begin{array}{c}\text { Código } \\
\text { de muestra }\end{array}$} & $\varepsilon_{\text {máx }}$ medida & $\varepsilon_{\text {máx }}$ láser \\
\hline & & $\begin{array}{l}\text { Elongación } \\
\text { máxima por } \\
\text { método físico }\end{array}$ & $\begin{array}{l}\text { Elongación } \\
\text { máxima } \\
\text { por método } \\
\text { analítico }\end{array}$ \\
\hline 1 & M2268-14-1 & 5.06 & 4.30 \\
\hline 2 & M2268-14-10 & 2.16 & 2.75 \\
\hline 3 & M2268-14-11 & 5.00 & 3.29 \\
\hline 4 & M2268-14-12 & 4.62 & 4.25 \\
\hline 5 & M2268-14-13 & 1.71 & 1.50 \\
\hline 6 & M2268-14-15 & 3.75 & 1.50 \\
\hline 7 & M2268-14-16 & 2.32 & 2.26 \\
\hline 8 & M2268-14-17 & 4.23 & 3.50 \\
\hline & Promedio & 3.61 & 2.92 \\
\hline & iación Estándar & 1.35 & 1.11 \\
\hline
\end{tabular}

\begin{tabular}{|c|c|c|c|c|c|c|}
\hline Muestra & $\begin{array}{c}\text { Hipótesis nula } \\
\mathbf{H}_{0}\end{array}$ & $\begin{array}{c}\text { Confianza } \\
(\%)\end{array}$ & $\begin{array}{c}\text { Estadístico } \boldsymbol{F}_{\mathrm{t}} \\
\text { de tabla }\end{array}$ & $\begin{array}{c}\text { Estadístico } \mathbf{f}_{0} \\
\text { calculado }\end{array}$ & Comparación & Interpretación \\
\hline M2267-14 (5,25mm) & \multirow{2}{*}{$\varepsilon_{\mathrm{u}(\mathrm{med})}=\varepsilon_{\mathrm{u}(\text { laser })}$} & 95.0 & 4.60 & 0.69 & $F_{t}>F_{c}$ & Se acepta Ho \\
\hline M2268-14 $(6,35 \mathrm{~mm})$ & & 95.0 & 4.60 & 3.06 & $F t>F c$ & Se acepta Ho \\
\hline
\end{tabular}

\begin{tabular}{|c|c|c|c|c|c|c|}
\hline Muestra & $\begin{array}{c}\text { Hipótesis nula } \\
\mathbf{H}_{0}\end{array}$ & $\begin{array}{c}\text { Confianza } \\
(\%)\end{array}$ & $\begin{array}{c}\text { Estadístico } \boldsymbol{F}_{\mathrm{t}} \\
\text { de tabla }\end{array}$ & $\begin{array}{c}\text { Estadístico } \mathbf{f}_{0} \\
\text { calculado }\end{array}$ & Comparación & Interpretación \\
\hline M2267-14 (5,25mm) & \multirow{2}{*}{$\mathbf{E}_{\mathrm{ext}}=\mathbf{E}_{\text {laser }}$} & 95.0 & 4.60 & 0.79 & $F_{t}>F_{c}$ & Se acepta Ho \\
\hline M2268-14 (6,35mm) & & 95.0 & 4.60 & 0.28 & $F t>F_{C}$ & Se acepta Ho \\
\hline
\end{tabular}

\begin{tabular}{|c|c|c|c|c|c|c|}
\hline Muestra & $\begin{array}{c}\text { Hipótesis nula } \\
\mathbf{H}_{0}\end{array}$ & $\begin{array}{c}\text { Confianza } \\
(\%)\end{array}$ & $\begin{array}{c}\text { Estadístico } \boldsymbol{F}_{\mathrm{t}} \\
\text { de tabla }\end{array}$ & $\begin{array}{c}\text { Estadístico } \mathbf{f}_{0} \\
\text { calculado }\end{array}$ & Comparación & Interpretación \\
\hline M2267-14 (5,25mm) & \multirow{2}{*}{$f_{y(\text { ext })}=f_{y(\text { laser })}$} & 95.0 & 4.60 & 0.99 & $F_{t}>F_{c}$ & Se acepta Ho \\
\hline M2268-14 (6,35mm) & & 95.0 & 4.60 & 0.34 & $F t>F C$ & Se acepta Ho \\
\hline
\end{tabular}




\subsubsection{Análisis del Módulo de elasticidad}

$\mathrm{Al}$ igual que con el valor de elongación, a los resultados obtenidos del Módulo de elasticidad, se prueba la hipótesis nula, obteniéndose los siguientes resultados: (ver tabla 11 en pagina 33 ).

$\mathrm{Al}$ igual que con la propiedad anterior, la hipótesis nula propuesta no se rechaza. Esto implica que los valores de Módulo de elasticidad, calculados a partir de los datos reportados por el extensómetro láser, cuantitativamente no existen diferencias significativas con respecto a los calculados con el extensómetro de contacto. Es decir, que mediante este análisis estadístico se valida, con un $95 \%$ de nivel de confianza, el uso del extensómetro láser para determinar el Módulo elasticidad a partir de ensayos de tensión.

\subsubsection{Análisis del esfuerzo de fluencia}

A los datos del esfuerzo de fluencia, también se les aplicó la prueba de hipótesis nula, para determinar la validez de los resultados obtenidos utilizando el extensómetro láser. Los resultados obtenidos son los siguientes:

(ver tabla 12 en pagina 33 ).

Al igual que en los casos anteriores, la hipótesis nula propuesta no se rechaza. Por lo tanto, es posible aseverar con un $95 \%$ de nivel de confianza que, los valores de esfuerzo de fluencia obtenidos mediante el extensómetro láser y de contacto no presentan diferencias significativas. Lo que implica que es posible utilizar el extensómetro láser para la determinación de esta propiedad en el ensayo de tensión.

\section{CONCLUSIONES}

A partir de la observación y análisis de los diagramas de esfuerzo-deformación, es posible concluir que los alambres de ambos diámetros mostraron un comportamiento que se adapta al esperado teóricamente. Además, este análisis permite evidenciar que las propiedades mecánicas calculadas con base en las mediciones de extensómetros láser y de contacto son similares.

Del análisis estadístico de esfuerzo de fluencia, esfuerzo máximo y Módulo de elasticidad, se concluye con un 95\% de nivel de confiabilidad, que no existen diferencias significativas entre los resultados obtenidos mediante el uso del extensómetro de contacto y el láser. Por lo tanto, es posible afirmar que el uso del extensómetro láser reproduce datos confiables, permitiendo su utilización para determinar las propiedades mecánicas de los alambres de acero.

Por último, se concluye que el método de obtención y análisis aplicado en el estudio es válido para el estudio de materiales con regiones de fluencia que no están claramente definidas.

\section{REFERENCIAS}

Abarca, A; Monge, A. Comparación de determinación de propiedades mecánicas en barras de refuerzo para concreto con extensómetro axial convencional y extensómetro láser, Revista Métodos \& Materiales, pp $4-20$. Vol 3, 2013.

ASTM A1064 / A1064M - 13. (2013). Standard Specification for CarbonSteel Wire and Welded Wire Reinforcement, Plain and Deformed, for Concrete. ASTM International, West Conshohocken, PA, 2013, DOI: 10.1520/A1064_A1064M. www.astm.org.

ASTM A370 / A370 - 14. (2014). Standard Test Methods and Definitions for Mechanical Testing of Steel Products. ASTM International, West Conshohocken, PA, 2013, DOI: 10.1520/A0370-14. www.astm.org.

ASTM E83 / E83-10. (2010). Standard Practice for Verification and Classification of Extensometer Systems. ASTM International, West Conshohocken, PA, 2010, DOI: 10.1520/E0083-10A. www.astm.org.

Avner, S. (1988). Introducción a la metalurgia física. 2da ed. Mc Graw Hill/ Interamericana de México. D.F. México.

Gere, J. Timoshenko, S. (1986). Mecánica de Materiales. International Thomson. D.F. México.

MTS Systems Corporation. (2013) Services and Accessories Catalog. Estados Unidos. www.mts.com

MTS Systems Corporation (2009). Series LX Laser Extensometer Product Information. Manual usuario: 015-207-801 F, Estados Unidos www. mts.com

Navarro, M (2010). Probabilidad y Estadística: un enfoque teórico y práctico. Editorial Tecnológica de Costa Rica. Instituto Tecnológico de Costa Rica. Cartago. Costa Rica.

Reglamento Técnico RTCR 452:2011 Barras y Alambres de Acero de refuerzo para concreto. $N^{\circ}$ 37341-MEIC. La Gaceta Digital 11/10/2013 $\mathrm{N}^{\circ} 152$. Recuperado de: http://www.nacion.com/archivo/ReglamentoRTCR-Alambres-Refuerzo-Concreto_LNCFIL2 0131119_0002.pdf Smith, W. Hashemi, J. (2006) Fundamentos de la ciencia e Ingeniería de materiales. 4ta Ed. Mc Graw Hill. D.F. México.

Smith, W. Hashemi, J. (2006) Fundamentos de la ciencia e Ingeniería de materiales. 4ta Ed. Mc Graw Hill. D.F. México. 